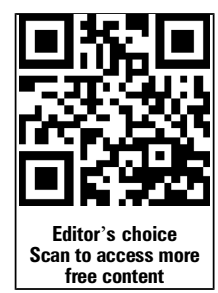

${ }^{1}$ Drug Safety Center, Leipzig University, Leipzig, Germany ${ }^{2}$ Department of Clinical Pharmacy, Leipzig University, Leipzig, Germany ${ }^{3}$ Pharmacy Department of the University Hospital Leipzig, Leipzig, Germany ${ }^{4}$ Department of Orthopaedic Surgery, University Hospital Leipzig, Leipzig, Germany

\section{Correspondence to} Professor Thilo Bertsche Department of Clinical Pharmacy, Leipzig University, Eilenburger Str. 15a, Leipzig 04317, Germany;

klinische.pharmazie@unileipzig.de

Received 29 August 2014 Revised 23 October 2014 Accepted 17 November 2014 Published Online First 10 December 2014

\section{CrossMark}

To cite: Schiek $S$, Hildebrand C, Ranft D, et al. Eur J Hosp Pharm 2015:22:156-160.

\title{
A cohort study investigating medication management by pharmacists to prevent drug-related problems in pain therapy
}

Susanne Schiek, ${ }^{1,2}$ Carolin Hildebrand, ${ }^{2,3}$ Donald Ranft, ${ }^{3}$ Axel Dürrbeck, ${ }^{3}$ Mohamed Ghanem, ${ }^{4}$ Georg von Salis-Soglio, ${ }^{4}$ Roberto Frontini, ${ }^{1,3}$ Thilo Bertsche ${ }^{1,2}$

ABSTRACT
Objectives We aimed at identifying drug-related problems in pain therapy and assessed the effect of medication management by pharmacists in routine care. Methods We performed a cohort study consisting of a control (no pharmaceutical service) and a medication management group receiving pharmaceutical services including recommendations to physicians. We defined pharmacotherapeutic and administrative drug-related problems in pain therapy and analysed patient charts to assess the outcomes. Active participants in the study were not involved in study assessment. Outcomes were the number of patients and the number of analgesic prescriptions with drug-related problems.

Results We analysed 414 medication records (controls: 208, medication management: 206). Our intervention had no influence on the number of patients with drugrelated problems following analgesic prescriptions (control: 166/208, 80\% vs medication management: 155/206, 75\%, $p=0.266)$. In 98 control group patients $(47 \%)$, we found at least four drug-related problems. This fraction was smaller in the medication management group, with 66 patients ( $32 \% ; p=0.002)$. The number of analgesic prescriptions leading to drug-related problems decreased from controls with $381 / 627$ (61\%) to $308 /$ $654(47 \%)$ in the medication management group $(p<0.001)$.

Conclusions Current pain therapy standards were not strictly adhered to in routine analgesic prescriptions for orthopaedic patients. The inclusion of pharmacists into the team successfully decreased drug-related problems. However, further interventions are required to achieve optimal effects.

\section{INTRODUCTION}

Nowadays, pharmacists in the ward and in the backup office engage in a broad range of quality-related and patient-related aspects of individualised therapy. ${ }^{1}{ }^{2}$ The inclusion of pharmaceutical knowledge into an interdisciplinary team contributes to better clinical outcomes. Drug-drug interaction management, ${ }^{3}$ drug dosing in patients with renal insufficiency ${ }^{4}$ and special pharmaceutical issues such as incompatibilities ${ }^{5}$ are examples of areas in which successful interventions enhance quality improvement. Furthermore, pharmacists collaborate with physicians, nurses and other healthcare providers in order to achieve lower mortality rates and higher cost efficiency. ${ }^{6} 7$ Yet, pain therapy remains an area in which collaboration of pharmacists and physicians in an interdisciplinary team is rarely reported. Especially, intervention studies investigating the benefit of such collaborations are widely missing.

Severe pain strongly impairs the quality of life. ${ }^{8}$ Patients fear pain or face barriers to pain management while undergoing surgery in hospital. ${ }^{9}$ Therefore, analgesic therapy, one of the important pillars of pain management, should be of special concern in quality improvement strategies. The role of pharmacists has already been discussed with regard to particular areas of pain therapy such as opioid overprescribing. ${ }^{10}$ Positive effects of educational interventions that pharmacists delivered to patients with chronic pain have been reported. ${ }^{11}$ Moreover, in outpatients receiving palliative care, pharmacists have been shown to contribute to optimised pain therapy and symptom control. ${ }^{12}$ As previously reported, ${ }^{13}$ multidisciplinary pain management including interventions by pharmacists increases guideline adherence and even improves the patients' individual pain situation. In summary, the literature has mainly dealt with chronic pain in patients with tumour so far.

Recently, nationwide guidelines have been published addressing, for example, perioperative acute pain management. ${ }^{14}$ These guidelines recommend best practices of pain therapy including pain assessment and evidence-based treatment in general and with a focus on surgical procedures. Although these guidelines give advice on how to handle practical aspects of pain therapy, their implementation has not been fully completed yet. ${ }^{15}$ This situation is associated with an elevated risk of drug-related problems. Therefore, we aimed at investigating whether drug-related problems occurred in orthopaedic patients in a hospital setting with routine prescriptions of analgesics (control group). We hypothesised that a comprehensive pharmaceutical service would influence the prevalence of drug-related problems in pain therapy (medication management group).

\section{MATERIALS AND METHODS \\ Participants and setting}

Patients on three orthopaedic wards with 49 beds at our university hospital were eligible for the study. In specific pain situations, physicians in the ward could request consultative examination by pain therapists or an acute pain service, a structured programme by pain nurses under supervision of pain therapists. An internal standard for pain therapy, however, did not exist at the time of this study. 


\section{Study design}

We performed a cohort study consisting of a control (without pharmaceutical service) and a medication management group (with pharmaceutical service). We included patients who were hospitalised in the participating wards during the respective study periods. In both the groups, patients were included consecutively, only once and only during their first hospital stay within the study period. All control group patients were discharged before any pharmaceutical service was implemented. The first period (control group, 9 weeks) started in May 2010 and the second study period (medication management group, 10 weeks) in May 2011. The medication management group received comprehensive medication management provided by pharmacists. This structured service was prospectively planned and implemented in July 2010. Four pharmacists with several years of experience in clinical pharmacy provided this service. It was offered within routine care for all newly hospitalised patients and, additionally, once a week for all patients in the wards included. The pharmacists assessed the adequacy of the patients' medication including parameters such as dosing, formulation and drug-drug interactions. On completing the medication management, pharmacists informed physicians about their recommendations (eg, modification of the medication profile or monitoring of laboratory data) in a written form.

\section{Study assessment}

We predefined drug-related problems with analgesic prescriptions (including co-analgesics). Active participants in the study were not involved in study assessment. Drug-related problems were defined as deviations from nationwide guidelines for pain therapy, ${ }^{14}$ from WHO standards, ${ }^{16}$ from the Summary of Product Characteristics, from drug information services ${ }^{17}$ and from the current literature about medication errors and their evaluation. ${ }^{18} 19$ We classified drug-related problems into pharmacotherapeutic or administrative problems. We analysed analgesic-related data gained from patient charts to assess therapy adequacy with respect to predefined drug-related problems. For the assessment of prevalence, we considered the prescription in the last version of the patient chart before discharge and analysed analgesics prescribed to patients during their hospital stay. Patients were not included in our study in those cases where their charts were not available. We analysed the data after the study periods had finished. This ensured that any influence the data evaluation might have had on the pharmacists participating in the study was excluded.

\section{OUTCOMES AND POWER CALCULATION}

We assessed the number of patients (primary outcome) and the number of analgesic prescriptions with at least one drug-related problem. The prevalence of those problems per prescription, per patient, in total, within predefined categories, and the number of prescribed analgesics were further aspects we recorded (secondary outcomes). According to the results of a previous study regarding tumour pain ${ }^{13}$ and pilot investigations in acute pain settings, we hypothesised that without medication management at least $50 \%$ of patients would be affected by at least one drug-related problem in pain categories. A decrease by at least 15\% (as compared with the control group, ie, a rate of $\leq 35 \%$ in the medication management group) was considered clinically relevant. Assuming rates within the mentioned range, a double-sided test at a significance level of $\alpha=0.05$ and a sample size of at least 167 patients will provide a power of $1-\beta=0.80$. We presented data as median with the first and third quartile or as frequencies of the respective patients or prescriptions, as appropriate. Results were analysed by $\chi^{2}$ test or Mann-Whitney U test, as appropriate. Patient characteristics were analysed to ensure comparability of patient groups and to exclude potential result-modifying factors. In the analysis of secondary outcomes, we made no adjustment for multiple testing. A p value of $<0.05$ was considered significant. Calculations were conducted using SPSS (Statistical Package for the Social Science, V.20, IBM, USA).

\section{RESULTS}

Altogether, 478 consecutive patients were hospitalised in the participating wards during the study periods. A medication profile was available for 414 of those patients, of which 208 patients were in the control and 206 in the medication management group. Patients were mainly hospitalised for surgical interventions (59\% in controls vs $65 \%$ in the intervention group) or conservative orthopaedic treatment including pain therapy (41\% vs 35\%). Patient characteristics did not differ between the two groups (table 1). Participating physicians $(n=21$, including six senior physicians) were orthopaedics with a professional experience ranging from 1 to 37 years. Physicians prescribed a median of three analgesics (Q25/Q75:2/4) per patient, in both the control and the medication management group (n.s.). We analysed 627 analgesic prescriptions by physicians in the control group and 654 in the medication management group. Apart from WHO-II opioids, which were less frequently prescribed in the medication management group $(p=0.002)$, prescription

Table 1 Patient characteristics

\begin{tabular}{|c|c|c|c|}
\hline & $\begin{array}{l}\text { Control } \\
\text { group }\end{array}$ & $\begin{array}{l}\text { Medication } \\
\text { management } \\
\text { group }\end{array}$ & p Value \\
\hline Patients (n) & 208 & 206 & \\
\hline Median age (Q25/Q75) (years) & $64(48 / 71)$ & $63(51 / 72)$ & 0.375 \\
\hline Females (n (\%)) & $112(54)$ & $128(62)$ & 0.088 \\
\hline Median weight (Q25/Q75) (kg) & $76(67 / 87)$ & $75(68 / 88)$ & 0.997 \\
\hline $\begin{array}{l}\text { Median hospital stay in total } \\
\text { (Q25/Q75) (days) }\end{array}$ & $7(5 / 11)$ & $7(5 / 14)$ & 0.579 \\
\hline $\begin{array}{l}\text { Patients undergoing surgery } \\
(\mathrm{n}(\%)) \text {, of which }\end{array}$ & $122(59)$ & $134(65)$ & 0.181 \\
\hline Spine surgery (n (\%)) & $33(16)$ & $46(22)$ & 0.094 \\
\hline Endoprosthetic surgery (n (\%)) & $26(13)$ & $19(9)$ & 0.284 \\
\hline Foot surgery (n (\%)) & $21(10)$ & $12(6)$ & 0.109 \\
\hline Knee surgery (n (\%)) & $15(7)$ & $26(13)$ & 0.065 \\
\hline Shoulder surgery (n (\%)) & $13(6)$ & $19(9)$ & 0.257 \\
\hline Hand surgery (n (\%)) & $7(3)$ & $5(2)$ & 0.596 \\
\hline Other surgery (n (\%)) & 7 (3) & $7(3)$ & 1.000 \\
\hline $\begin{array}{l}\text { Median hospital stay after } \\
\text { surgery (Q25/Q75) (days) }\end{array}$ & $8(4 / 13)$ & $7(4 / 14)$ & 0.807 \\
\hline $\begin{array}{l}\text { Patients undergoing conservative } \\
\text { orthopaedic treatment }(\mathrm{n}(\%))\end{array}$ & $41(20)$ & $35(17)$ & 0.475 \\
\hline $\begin{array}{l}\text { Patients undergoing specific } \\
\text { orthopaedic pain treatment } \\
\text { including minimally invasive } \\
\text { techniques }(\mathrm{n}(\%))\end{array}$ & $45(22)$ & $37(18)$ & 0.348 \\
\hline $\begin{array}{l}\text { Patients with acute pain service } \\
\text { (n (\%)) }\end{array}$ & $20(10)$ & $25(12)$ & 0.410 \\
\hline $\begin{array}{l}\text { Patients with consultative } \\
\text { examination by pain therapists } \\
\text { (n (\%)) }\end{array}$ & $15(7)$ & $13(6)$ & 0.715 \\
\hline $\begin{array}{l}\text { Patients with } \mathrm{Cl}<60 \mathrm{~mL} / \mathrm{min} \\
\text { (n (\%)) }\end{array}$ & $23(11)$ & $25(12)$ & 0.732 \\
\hline
\end{tabular}


behaviour concerning other analgesics remained comparable in the two groups (table 2).

Our medication management had no influence on the number of patients with at least one drug-related problem with analgesic prescriptions (controls: 166/208, 80\% vs medication management: $155 / 206,75 \%, p=0.266)$. In $98(47 \%)$ of the control group patients we found at least four drug-related problems. This fraction decreased to $66(32 \% ; \mathrm{p}=0.002)$ patients in the medication management group.

With $381 / 627(61 \%)$, the number of analgesic prescriptions with at least one drug-related problem was lower in the control group than in the medication management group with 308/654 $(47 \% ; \mathrm{p}<0.001)$. The rate of drug-related problems/prescription decreased from 1.77 by $31 \%$ to 1.22 (relative risk reduction (RRR); $\mathrm{p}<0.001)$, and notably, prescriptions with 3 and $\geq 4$ drug-related problems decreased significantly $(p=0.006$ and $\mathrm{p}<0.001$; figure 1).

Regarding pharmacotherapeutic drug-related problems, analgesics in problematic 'pain cocktails' and 'opioids in inappropriate combination' were significantly less frequently prescribed in the medication management group as compared with controls $(\mathrm{p}=0.005$ and $\mathrm{p}=0.041$, table 3$)$. Other subgroups in this area remained without statistically significant alteration. Among administrative drug-related problems, several subcategories such as incomplete information concerning single dose, dosage instruction (eg, dosing interval), daily dose and drug name or dosage form improved (each $\mathrm{p}<0.001$ ).

\section{DISCUSSION}

We found that, based on current pain standards, drug-related problems in analgesic prescriptions were alarmingly frequent, with $80 \%$ of orthopaedic patients being affected in controls. This indicates that strategies for quality improvement in this field are absolutely necessary. We prevented drug-related problems in pain therapy by involving pharmacists in the multidisciplinary team.

Due to the interventions, prescriptions with drug-related problems moderately but significantly decreased from $61 \%$ to $47 \%$. Mainly prescriptions with three and more drug-related problems declined, and prescriptions without any drug-related problem reached more than $50 \%$ in the medication management group.

Table 2 Prescribed analgesics, with 627 analgesics prescribed in the control group and 654 in the medication management group

\begin{tabular}{|c|c|c|c|}
\hline $\begin{array}{l}\text { Class of } \\
\text { analgesics }\end{array}$ & $\begin{array}{l}\text { Control group } \\
\text { (n/total; }(\%))\end{array}$ & $\begin{array}{l}\text { Medication } \\
\text { management group (n/ } \\
\text { total; }(\%))\end{array}$ & $p$ Value \\
\hline $\begin{array}{l}\text { Opioid } \\
\text { analgesics* }\end{array}$ & 240/627 (38) & 227/654 (35) & 0.363 \\
\hline WHO-II opioids & $125 / 627(20)$ & $89 / 654(14)$ & 0.002 \\
\hline WHO-III opioids & $115 / 627$ (18) & $138 / 654(21)$ & 0.215 \\
\hline $\begin{array}{l}\text { Non-opioid } \\
\text { analgesics* }\end{array}$ & $406 / 627(65)$ & $407 / 654(62)$ & 0.349 \\
\hline NSAID & $136 / 627(22)$ & $135 / 654(21)$ & 0.646 \\
\hline Coxibs & $17 / 627(3)$ & $13 / 654(2)$ & 0.392 \\
\hline Dipyrone & $163 / 627(26)$ & $158 / 654(24)$ & 0.448 \\
\hline Paracetamol & $72 / 627(12)$ & $80 / 654(12)$ & 0.679 \\
\hline Flupirtine & 18/627 (3) & 21/654 (3) & 0.723 \\
\hline Co-analgesics & $50 / 627(8)$ & $62 / 654(10)$ & 0.340 \\
\hline
\end{tabular}

This is an important but not sufficient step to improve pain therapy. Especially patients suffering from a large number of drug-related problems benefitted from the medication management. However, the number of patients with at least one drug-related problem with analgesic prescriptions remained unaffected by our intervention. This indicates a need for a more frequent medication management.

Our once-a-week service addressed the individual patients' pain medication rarely more than once during their hospital stays. Pain therapy should regularly be tailored to the individual pain situation. Hence, if pharmacists participate in pain therapy management, they should be involved in a daily ward round or patient chart analysis to reach an optimised benefit. Within daily service, pharmacists could give recommendations after any change of medication or in the clinical situation. They could check if their recommendations are put into practice and, if necessary, repeat these recommendations. Optimising the implementation as well as reducing communication and documentation problems should even improve the positive outcomes.

Our study was restricted to the assessment of drug-related problems (predefined standards) and did not assess the actual pain of patients. A multicentre study by Vallano et $a l^{20}$ reported that pain prevalence was higher in those patients in whom analgesic treatment did not adhere to guidelines. Therefore, the high prevalence of drug-related problems in our study indicates insufficient pain control in our patients. Thus, under the medication management, the prevalence of drug-related problems decreased respectably by an RRR of nearly one-third. Our results support the point of view that pharmacists play an important role in the interdisciplinary team by supporting physicians in a broad range of therapies. ${ }^{21}$

Physicians quite commonly prescribed analgesics in problematic 'pain cocktails', which frequently were combinations of dipyrone, tramadol and metoclopramide. Mainly because of their inconsistent quantitative composition and unclear stability, experts consider such combinations obsolete. Due to medication management, physicians prescribed 'pain cocktails' less frequently, with a decrease of $41 \%$.

A further positive outcome might be that WHO-II opioids were prescribed less frequently in the medication management group.

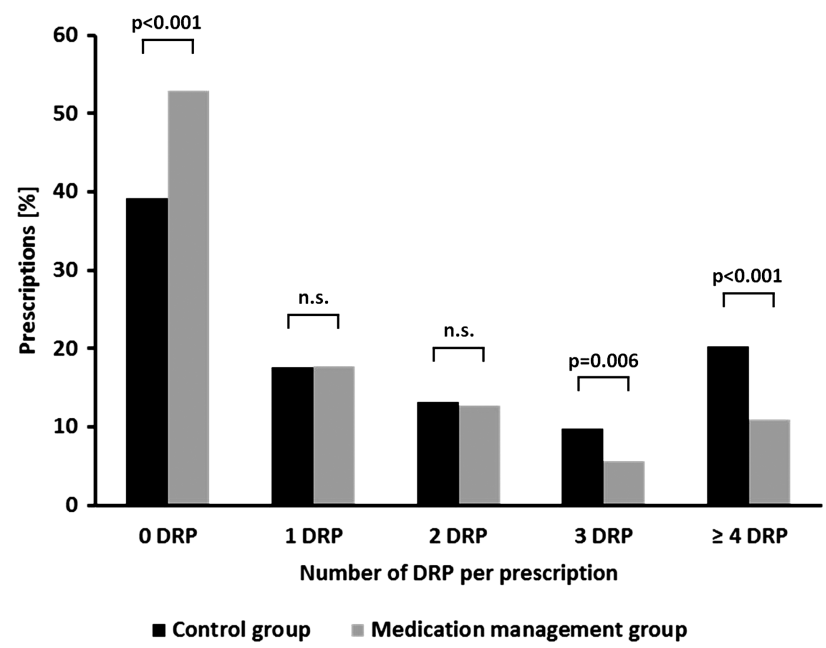

Figure 1 Distribution of prescriptions in relation to the number of drug-related problems (DRP) per prescription in the control group (black, total of 627 prescriptions) and in the medication management group (grey, total of 654 prescriptions). 


\section{Pharmacotherapeutic drug-related problems}

Non-opioid in inappropriate combination (eg, two different NSAIDs)

Opioid in inappropriate combination (eg, WHO-II with WHO-III opioid)

Opioid without non-opioid (although indicated)

Overdose (eg, exceeding dose limits of paracetamol)

Inappropriate administration instruction (eg, dosing interval differs from SmPC)

Missing or inappropriate supportive therapy (eg, missing laxatives)

Analgesic in inappropriate dosage form (eg, opioids with sustained release on demand or immediate release in fixed dose)

Analgesics in problematic 'pain cocktails' (eg, of tramadol, dipyrone and metoclopramide prescribed in a fixed

but not specified way as 'pain cocktail')

Drug-drug interactions

Administrative drug-related problems

Duplicate prescriptions of analgesics

Prescription of analgesic with incomplete information concerning single dose

Prescription of analgesic with incomplete information concerning dosage instruction (eg, dosing interval)

Prescription of analgesic with incomplete information concerning daily dose

$\begin{array}{ccr}14 / 406(3)^{* *} & 18 / 407(4)^{* *} & 0.475 \\ 139 / 240(58)^{* * *} & 110 / 227(49)^{* * *} & 0.041 \\ 11 / 240(5)^{* * *} & 16 / 227(7)^{* * *} & 0.254 \\ 15 / 627(2) & 8 / 654(1) & 0.115 \\ 14 / 627(2) & 16 / 654(2) & 0.800 \\ 82 / 627(13) & 70 / 654(11) & 0.189 \\ 13 / 627(2) & 14 / 654(2) & 0.933 \\ & & \\ 68 / 627(11) & 42 / 654(6) & 0.005 \\ & & 0.509 \\ 84 / 627(13) & 96 / 654(15) & 0.863 \\ 32 / 627(5) & 32 / 654(5) & <0.001 \\ 165 / 627(26) & 70 / 654(11) & <0.001 \\ 185 / 627(30) & 126 / 654(19) & <0.001 \\ 204 / 627(33) & 131 / 654(20) & <0.001 \\ 81 / 627(13) & 47 / 654(7) & \end{array}$

Prescription of analgesic with incomplete information concerning drug name or dosage form

$81 / 627(13)$

$47 / 654(7)$

Based on the total number ${ }^{*}$ ) of all analgesic prescriptions, if not otherwise specified; ${ }^{* *}$ ) of non-opioid analgesic prescriptions; ${ }^{* *}$ ) of opioid analgesic prescriptions.

NSAIDs, non-steroidal anti-inflammatory drugs; SmPC, Summary of Product Characteristics.

Identified drug-related problems may result in avoidable adverse effects because analgesics are among the drugs with significant harm potential. $^{22}$ Non-steroidal anti-inflammatory drugs (NSAID) in particular, possess increased end organ toxicity and may further reduce perioperatively reduced renal function. They also inhibit thrombocyte function, leading to an increased risk of bleeding. Especially in the elderly, as in our patient group, NSAIDs pose a particular risk of causing gastric ulcer. ${ }^{23}$ Opioids, on the contrary, can lead to adverse events that go along with a reduced quality of life. Constipation and vomiting, in particular, are among the most frequent adverse events requiring pharmacotherapy with supportives such as laxatives or antiemetics. $^{24}$ In this study, however, the medication management did not significantly improve supportive care. Thus, we identified this point as one of the key aspects for further interventions.

The high prevalence of drug-related problems, which was reduced due to the medication management, underlines the need to further involve pharmacists in interdisciplinary teams. Opioids were identified as a particular target of educational interventions. ${ }^{25}$ Inclusion of pharmacists and co-working with physicians have already been implemented in primary chronic pain management. ${ }^{26-29}$ However, apart from counselling healthcare providers, future pharmaceutical strategies should also address the patient directly. ${ }^{30}$

It is a special strength of this study that active participants in the study were not involved in study assessment. Thus, we excluded any influence of the involved pharmacists on the assessment of their interventions. This is in contrast to a former study ${ }^{13}$ in which the intervening investigators assessed their own effects. Moreover, we did not assess the prevalence of interventions addressed by pharmacists in this evaluation but the number of drug-related problems identified by an independent review of the patient charts. This way, we investigated whether drug-related problems were resolved and whether modifications of the medication management were actually put into practice.

\section{Limitations}

While analgesic overdosing was not a major problem in our study, analgesic underdosing and inadequate pain treatment might be a result of missing information about pain intensity. Pain and pain intensity were not routinely documented on the wards involved. As a consequence of these findings, documentation of pain intensity will be implemented and investigated in a further project. It should be taken into account that structured pain assessment influences pain therapy even in the control group without additional intervention and that, therefore, pain

\section{Key messages}

What is already known on this subject?

- Including pharmacists in the interdisciplinary team contributes to better outcomes in many medical fields.

- Pain therapy is a typical field for interdisciplinary teamwork. Yet, pharmacists are not regularly involved in routine pain management so far.

- Recently, guidelines for perioperative acute pain management have been published but their implementation in routine practice remains unclear.

- Studies investigating the contribution of pharmacists to improving guideline adherence in perioperative pain therapy and to solving drug-related problems, caused by insufficient guideline adherence, are still missing.

\section{What this study adds?}

- We found that drug-related problems in pain therapy were alarmingly frequent in routine care.

- Involving pharmacists on a weekly basis contributes to a substantial improvement in following pain standards for orthopaedic patients. 
assessment itself is, in itself, an intervention within the total quality process of pain treatment. ${ }^{13}$

Even though we are not aware of any specific confounder or bias, we cannot completely exclude modifying factors due to the non-randomised study design. To minimise these effects, we chose the same study units in controls and in the medication management group in order to assess the same staff and to ensure verified comparability of the two patient groups. Additionally, we chose the same time of the year for the two study periods to avoid seasonal impact.

\section{Conclusion}

We identified pain in orthopaedic patients as an important field for quality improvement strategies in routine care. Pharmacists support analgesic prescribing practices by physicians in a collaborative manner considering administrative and pharmacotherapeutic drug-related problems. Our medication management once a week might be a pragmatic but successful instrument transferable to other settings with limited resources. However, intensified interventions (eg, with daily feedback to physicians) are required to achieve the full benefit for all patients.

Acknowledgements We would like to thank all participating pharmacists, physicians and nurses on the ward for their kind support. We also thank PD Dr Astrid Bertsche and Sabine Budnick for proofreading our manuscript.

Funding Susanne Schiek was co-financed by the Saxony Chamber of Pharmacist.

\section{Competing interests None.}

Ethical approval The Ethics Committee at the Medical Faculty of Leipzig University approved the study (approval no. 384-11-12122011).

Provenance and peer review Not commissioned; externally peer reviewed.

\section{REFERENCES}

1 Rothschild JM, Churchill W, Erickson A, et al. Medication errors recovered by emergency department pharmacists. Ann Emerg Med 2010;55:513-21.

2 Klopotowska JE, Kuiper R, van Kan HJ, et al. On-ward participation of a hospital pharmacist in a Dutch intensive care unit reduces prescribing errors and related patient harm: an intervention study. Crit Care 2010;14:R174.

3 Bertsche T, Pfaff J, Schiller $P$, et al. Prevention of adverse drug reactions in intensive care patients by personal intervention based on an electronic clinical decision support system. Intensive Care Med 2010;36:665-72.

4 Bertsche T, Fleischer M, Pfaff J, et al. Pro-active provision of drug information as a technique to address overdosing in intensive-care patients with renal insufficiency. Eur J Clin Pharmacol 2009;65:823-9.

5 Bertsche T, Veith C, Stahl A, et al. A purging procedure for pantoprazole and 4-lumen catheters to prevent IV drug incompatibilities. Pharm World Sci 2010;32:663-9.

6 Bond CA, Raehl CL, Franke T. Clinical pharmacy services and hospital mortality rates. Pharmacotherapy 1999;19:556-4.

7 Avery AJ, Rodgers S, Cantrill JA, et al. A pharmacist-led information technology intervention for medication errors (PINCER): a multicentre, cluster randomised, controlled trial and cost-effectiveness analysis. Lancet 2012;379:1310-19.
8 Wang XS, Cleeland CS, Mendoza TR, et al. The effects of pain severity on health-related quality of life: a study of Chinese cancer patients. Cancer 1999;86:1848-55.

9 Yin $\mathrm{HH}$, Tse MM, Wong FK. Postoperative pain experience and barriers to pain management in Chinese adult patients undergoing thoracic surgery. J Clin Nurs 2012;21:1232-43.

10 Craig DS. The Pharmacists' Role in Patient-Provider Pain Management Treatment Agreements. J Pharm Pract 2012;25:510-16.

11 Bennett MI, Bagnall AM, Raine G, et al. Educational interventions by pharmacists to patients with chronic pain: systematic review and meta-analysis. Clin J Pain 2011;27:623-30.

12 Gagnon L, Fairchild A, Pituskin E, et al. Optimizing pain relief in a specialized outpatient palliative radiotherapy clinic: contributions of a clinical pharmacist. J Oncol Pharm Pract 2012;18:76-83.

13 Bertsche T, Askoxylakis V, Habl G, et al. Multidisciplinary pain management based on a computerized clinical decision support system in cancer pain patients. Pain 2009;147:20-8

14 Guideline on peri-operative pain management of the German Interdisciplinary Association for Pain Therapy [homepage on the Internet]. Bonn, Germany [cited 2014 July 23]. http://www.awmf.org/leitlinien.html

15 Wu CL, Raja SN. Treatment of acute postoperative pain. Lancet 2011;377:2215-25

16 World Health Organisation (WHO). Cancer pain relief [Internet]. 1996 [cited 2014 July 23]. http://whqlibdoc.who.int/publications/9241544821.pdf

17 Dosage adjustment to renal function. [updated 2014 May 23; cited 2014 July 23]. http://www.dosing.de

18 Hanlon JT, Schmader KE, Samsa GP, et al. A method for assessing drug therapy appropriateness. J Clin Epidemiol 1992:45:1045-51.

19 Dean B, Barber N, Schachter M. What is a prescribing error? Qual Health Care 2000;9:232-7.

20 Vallano A, Malouf J, Payrulet P, et al. Catalan Research Group for the Study of Pain in the Hospital. Analgesic use and pain in the hospital settings. Eur J Clin Pharmacol 2007;63:619-26.

21 Nkansah N, Mostovetsky O, Yu C, et al. Effect of outpatient pharmacists' non-dispensing roles on patient outcomes and prescribing patterns. Cochrane Database Syst Rev 2010;(7):CD000336.

22 Takata GS, Taketomo CK, Waite S.; California Pediatric Patient Safety Initiative. Characteristics of medication errors and adverse drug events in hospitals participating in the California Pediatric Patient Safety Initiative. Am J Health Syst Pharm 2008;65:2036-44.

23 Murakami K, Okimoto T, Kodama M, et al. Helicobacter pylori and NSAID-induced gastric ulcer in a Japanese population. J Gastroenterol 2009;44(Suppl 19):40-3.

24 Baldini A, Von Korff M, Lin EH. A review of potential adverse effects of long-term opioid therapy: a practitioner's guide. Prim Care Companion CNS Disord 2012;14: pii: PCC. $11 \mathrm{~m} 01326$.

25 Marlowe KF, Geiler R. Pharmacist's role in dispensing opioids for acute and chronic pain. J Pharm Pract 2012;25:497-502.

26 Li RM, Franks RH, Dimmitt SG, et al. Ideas and innovations: inclusion of pharmacists in chronic pain management services in a primary care practice. J Opioid Manag 2011;7:484-7.

27 Watts $\mathrm{G}$. Doctors told to collaborate with community pharmacists to improve pain management. BMJ 2012;344:e350.

28 Hadi MA, Alldred DP, Briggs $M$, et al. A combined nurse-pharmacist managed pain clinic: joint venture of public and private sectors. Int J Clin Pharm 2012;34:1-3.

29 Strickland JM, Huskey A, Brushwood DB. Pharmacist-physician collaboration in pain management practice. J Opioid Manag 2007;3:295-301.

30 Ryan N, Chambers C, Ralph C, et al. Evaluation of clinical pharmacists' follow-up service in an oncology pain clinic. J Oncol Pharm Pract 2013;19:151-8. 